\title{
Research on 3D Photography Technology and It's Applications in the Publishing Field
}

\author{
Yun Hong \\ Xi'an International University, Xi’an, Shaanxi, 710077, China
}

Keywords: 3D photography technology, 3D image, Publishing field.

\begin{abstract}
Under the background of the new round of the revolution of science and technology, modern publications are no longer confined to paper books, newspapers and magazines. The electronic books, pictures, audio and images have begun to appear. The applications of 3D photography in the field of publishing has changed the rigid and boring shortcomings of traditional publications, and has brought a new three-dimensional visual experience to readers. This paper analyzes the common technologies of 3D photography, and points out the applications of 3D photography in editing, printing, circulation and reading fields to provide some references for the relative researchers.
\end{abstract}

\section{Introduction}

The birth of the movie Avatar has brought a wave of 3D craze to the whole world, and brought infinite driving force for the development of 3D technology. 3D filmed two cameras to simulate human eyes, pictures of the left eye and the right eye. At present, the two cameras are arranged in two ways, one is horizontal and the other is vertically up and down. 3D photography is based on traditional photography technology, and uses the parallax of the left and right eyes to create and reproduce stereo vision images. Because there is a certain spacing distance between the eyes, watching the object, around the eyes to see the object has a certain angle difference, the human brain will see eye images together form the visual sense of space, sense of distance. This process is called perceived stereo vision 3D camera according to the principle of stereo vision, simulation using the 3D dual lens camera of the left eye, while shooting two pictures with angle difference, then the two images together through different viewing media or display media, makes people around the eyes. We can only see about the lens corresponds to the angle of the picture, in the picture to the human perception of three-dimensional space and distance, to reproduce stereo vision. 3D photography is divided into two parts: 3D photography and 3D image reproduction. It first uses the 3D camera to take pictures from about two observation points with an angle parallax, and then uses the 3D image reproduction technology to synthesize the photos taken by the camera. Because of the vision and depth of realistic space effect, 3D photography technology has been used in many fields of publishing and printing, commercial, construction, education, media, entertainment, military, physics, chemistry, astronomy, medical. Today, with the coexistence of traditional publishing and digital publishing, it is an important direction for publishing and communication to make traditional publishing a new life.

\section{Common Technologies of 3D Photography}

The technique of axial displacement effectively solves many problems such as X-ray deformation and clear focus in whole area. Because of the tilt, translation, and perspective control of the moving axis, the focal plane can be selected by adjusting the lens to correct the distortion produced by ordinary wide-angle lens taking. The translation and tilting functions of the lens are often used together, and the whole area focusing effect of the general photographic lens cannot be achieved while correcting the perspective distortion. In general, when shooting large buildings, to take a panoramic view of the building, the camera lens is made in the form of an overhead shot. As a result, because the camera focal plane has a certain angle to the main line of the tower, it causes perspective 
distortion, so the building in the picture appears to be falling. In this case, the shifting technique solves this problem by adjusting the translation and tilting functions of the moving axis, and the effect is obvious. Normally, the focal plane of the camera is parallel to the plane of the light-sensitive element, and the object on the focal plane must be clear and the defocus is blurry when taken with a large aperture. If you use a shift lens to break the focal plane, you can change the clear range. Knowing this principle, we can create the desired effect by moving the axis itself. One of the advantages is reflected in the axis lens in three-dimensional photography of the main performance of the prominent, the main picture of the sense of hierarchy division. With the 3D film in the last two years of another round of impact, the production process of modern film has also changed. The ordinary film to 3D film, is the area of different depth in the film by keying separation tracing way for different layers, and then again in the post production plane layer mapping to digital model or patch, set in software has two parallax cameras, respectively for rendering generation made. 3D movie in the history, there is a general film incomparable advantage. With the development of information technology, media material extends to the audience back to cinema has become many filmmakers are trying to study. To change the focal plane position through the original function principle translation tilt, to adjust the priorities of the screen, will be placed in the main body to a prominent position, the excess part or do not want to pay attention to things out false. In the traditional 3D stereo photography, we often find that to show a part of the main object, but inevitably will not want to shoot into the picture of other objects into, affecting the overall effect of the picture. Now, the introduction of moving axle in three-dimensional photography, the effect is self-evident.

\section{Applications of 3D Photography in the Publishing Field}

Editing Area. The editing technology based on 3D photography technology is mainly reflected in the image editing. The process of 3D publication image editing is basically the same as the editing process under the traditional model, but the processing methods are very different. 3D publications are mostly filmed using 3D shooting technology to save late processing time, such 3D image editing method is better than the later 3D image. Therefore, the editing methods and formats are different from those of traditional image processing. In addition, 3D publications read different from traditional reading, and general 3D reading requires reading with 3D glasses to achieve 3D effects. Do not wear any 3D device 3D reading, known as naked eye 3D reading, the current mainstream 3D publications on the market more need to wear 3D glasses, naked eye 3D technology is relatively expensive. The editor of the 3D publication is divided into two parts: text editing and image editing. The text editing of 3D publications is basically consistent with the editing process of traditional publications. In the edit image, there are two main techniques can save more post-processing time, and can get better 3D; the other is a complementary color stereo technology, the two images do complementary color processing, and incomplete overprint in the paper on. 3D publications currently have two types of reading: one is reading with 3D glasses, and the other is reading uncorrected 3D without any equipment. When making publications with 3D effects, the editing methods vary according to the publications reading the display medium. Production of newspapers and magazines, books and other print publications, the printing needs to be two images separated 3D pictures of different angles. The two picture is not completely overprinted together, and then by wearing 3D glasses, the reading of the 3D effect.

Printing Area. Although the printing and copying of publications based on 3D photography technology are basically the same as those of traditional publications, there are great differences in the process. Because 3D photography under the 3D publication is to show the 3D effect through the picture, so the printing needs to use high-quality coated paper and high temperature ink. 3D three-dimensional printing, this printing method is to use photosensitive material to complete, the picture is decomposed into pixels, combined with light canopy material, to achieve three-dimensional image effect. But compared to this type of printing, the former will increase the cost of printing, printing needs to use light canopy materials. Printing is an image reproduction in 3D photography, and the printing process of 3D publications is basically the same as that of traditional publications, 
and needs printing and printing. But in the printing process links are different, 3D publications mainly rely on pictures to show 3D visual effects, printing generally choose high-quality coated paper and high temperature ink, printed out after the gloss is good, not easy to fade. There are two main kinds of current can achieve the effect of 3D printing: one is red and blue two images separated 3D camera images into different angles, and then put these two pictures not completely overprinted together, through the red and blue glasses to view the 3D effect. In the printing process, because of the need to achieve the blue and red incomplete overprinted, the printing technology requirements are very high. In the printing process, people need to wear 3D glasses, always check the 3D effect, printing workload is relatively large. Another one is 3D printing technology, the image captured by the 3D camera, is decomposed into different angles and different levels of pixels, and recorded on a photosensitive material, combined with the grating material, a three-dimensional effect in the image plane. People do not need any tools to observe stereoscopic vision directly through their eyes. Due to the need for grating materials, the cost of printing will increase accordingly.

Circulation Area. Publishing is an important part of publishing and communication, which influences the development of the publishing industry. It is the source of the profits of publishing industry and the main means of publishing logistics. The model of publication and dissemination of 3D publications is not different from traditional publications. People are interested in 3D publications because they are interested in the visual effects of 3D, so the selling points of many works reflect the aspects of 3D photography and production. 3D publications in the market performance is very worthy of recognition. While readers are enjoying 3D readings, many readers think that $3 \mathrm{D}$ books have collections and the value of $3 \mathrm{D}$ is beginning to emerge. With the continuous improvement of the economic level and technology, the future publishing and dissemination will be dominated by 3D publishing, and the value of 3D publications will be gradually reflected. Due to the different arc lens refraction reflection distance, different space grating stereo images, requires a certain distance to watch shows the best effect. A three-dimensional printing ink needs to be bright and clean. Because any visible degree of foaming can affect image clarity and three-dimensional effect, the three-dimensional printing ink is not a foaming ink. The three-dimensional map publication will promote the expression of the map information from the development of traditional two-dimensional three-dimensional direction, providing an innovative form of expression for the map products, contribute to China's new products in the international market to map the map based on the traditional print stand out, help to enhance our map compilation and publication of the international influence and competitiveness in the international market, to improve China's surveying and mapping geographic information product application service system, comprehensively promote the surveying and mapping geographic information in various fields of social and economic application of the service capacity and the promotion of China's geographic information industry to promote the development of the international market and has important significance.

Reading Area. 3D publications can only reflect value through communication and audience acceptance. No matter which stereoscopic method is adopted, it is the vitality of 3D publications that is convenient for readers to accept. 3D publications currently have two different ways of reading, wearing 3D glasses to read publications, printing costs are low, but because the 3D glasses are filtered out a lot of color, so the color effect is not obvious. The publication cost of naked eye 3D reading is higher, especially the price of the electronic reader with naked eye 3D display function is higher, so it is difficult to popularize it. Traditional media has yet to find a new profit model. Although the reader provides an attractive opportunity to newspapers, publishers can save a huge amount of money in the printing and distribution, but the use of large screen readers to restore the traditional business model of charging readers, with advertising support, facing readers and doubts. Because even the iPad interface and no traditional newspaper layout are wide, advertising will drop substantially. Another obstacle is that some readers want to determine the price of their subscriptions and control their relationships with readers, which cannot be accepted by the media. Electronic readers let newspaper media open 3D new era. No matter what form of electronic reader, the future of newspapers will be dynamic, pictures, video and other new forms. Although it's like holding a real newspaper in hand, what you want to see and what you don't want to see is decided by your finger, but there will be a leap 
in the future. It must be 3D, dynamic and three-dimensional. Of course, to retain the form of the page, this is the media for the image processing of news communication, is an art creation. Now, the key is to make electronic readers as soon as possible readers of essential goods.

\section{Application Prospect of 3D Photography in the Publishing Field}

The use of 3D photography in publications enables publications to be superior to traditional publications in picture expressiveness and field appeal, and therefore has great room for development. The development of 3D photography, the 3D publications in a variety of ways, promote the development of publications of technology; the three is to promote the development of printing technology; four 3D publications visual effect is outstanding, the advertising revenue continues to improve, publishing revenue was five; 3D can achieve a variety of digital publications has promoted the reading effect. The development of new digital publishing. Every technology has a process from development to maturity, and the following problems exist in the application of 3D photography in the field of publishing and communication. You also need to edit the time of image processing technology and longer high 3D publications, is not conducive to a statute of limitations for the publication; compared with the traditional publications, 3D publications high requirement of printing technology and printing paper, the printing cost is also high. Consumption habits of readers also need to cultivate the reader, is still in the stage of 3D publications for curiosity, curiosity once weakened, the publications issued a greater impact; when the 3D photography technology in other such as movies, TV, Internet streaming media culture, virtual reality tours and other tourism industry, advertising and other commercial areas, as well as the culture, medicine, industry, architecture, cultural exhibitions, education, military, Internet, aerospace, archaeology, geography and other fields are widely used, and ultimately the formation of visual 3D industry chain mature, people demand for 3D publications will continue to increase, and the demand will promote the use of 3D technology in the field of publishing and communication more photography deep.

\section{Conclusion}

The applications of 3D technology in publishing field has indeed brought opportunities and changes to the traditional publishing industry. However, compared with the traditional publications, 3D photography is faced with the problems of the high cost, the complex editing and the long production cycle. These problems hinder the applications of 3D photography in the field of publishing, and the solution of these problems can popularize 3D publications in a large scale.

\section{References}

[1] Guan Bin. The Application of 3D HD Photography into 3D Data Construction of Museum-preserved Cultural Relics [J]. Journal of Guizhou University (Art Edition), 2015, 29(1): 66-70.

[2] Dai Zheng. Application of 3D Photography Technology in the Publishing Field [J]. Publishing Journal, 2014(5): 91-94.

[3] Li Guozhu, Xi Wenfei, Li Jingshan. 3D modeling of the cultural relics with UAV photography [J]. Journal of Suzhou University of Science and Technology (Natural Science), 2017, 34(2): 63-66.

[4] Niu Pengtao. On the Urban 3D Modeling Method Based on Oblique Photography Technique [J]. Value Engineering, 2014(26): 224-225. 